Construire l'image d'une personnalité politique à la télévision. Pygmalion Désemparé

\title{
Avant-Propos
}

\section{Odile Ausina}

\section{OpenEdition}

\section{Journals}

Édition électronique

URL : http://journals.openedition.org/edc/2880

DOI : $10.4000 /$ edc. 2880

ISSN : 2101-0366

\section{Éditeur}

Université de Lille

Édition imprimée

Date de publication : 1 mai 1987

Pagination : 29-38

ISSN : 1270-6841

Référence électronique

Odile Ausina, "Construire l'image d'une personnalité politique à la télévision. Pygmalion Désemparé », Études de communication [En ligne], 9 | 1987, mis en ligne le 08 juin 2013, consulté le 10 décembre 2020. URL : http://journals.openedition.org/edc/2880; DOI : https://doi.org/10.4000/edc.2880

Ce document a été généré automatiquement le 10 décembre 2020.

(c) Tous droits réservés 


\title{
Construire l'image d'une personnalité politique à la télévision. Pygmalion Désemparé
}

Avant-Propos

\author{
Odile Ausina
}

1 Pour éclairer la réflexion qui va suivre, rendons compte sommairement de quelques impressions de téléspectatrice plus que jamais subjective (magnétoscope, focalisation de l'attention, opinions préalables).

2 L'heure de vérité (octobre 87).

3 P. Méhaignerie sur la sellette. Comment servir Barre et Chirac? ... Il est mal à l'aise face au journaliste A. Duhamel qui insiste pour obtenir une réponse claire. Stratégies d'évitement. Moi,j'ai du mal à écouter.

Une idée toujours présente: "c'est terne». Physionomie, couleurs des vêtements (costume gris, chemise bleue pâle, dominante bordeaux de la cravate). Cette idée me rappelle Jospin dans une autre émission et dans "Questions à domicile» où même le contexte de l'appartement ne compensait pas ce «terne».

Mais ici, gestes, intonations, débit vont aussi dans le sens du flou et du partage entre deux positions peu compatibles.

Toujours par association d'idées je pense à la prestation de J. Lang au début de l'été 87. Je repasse la cassette. Celui que certains appellent " Jack le magnifique » crève l'écran...

Mince, costume bien porté frappé de couleurs vives (pochette, cravate), élégant dans la détente, gestes emphatiques, ronds sans molesse, large sourire dans un visage expressif, il est bien dans sa peau, en harmonie avec un discours clair, coloré d'images et de mots familiers mais sans affectation branchée.

Duhamel lui demande s'il n'a pas favorisé "des modes»? Il s'en défend, explique en quelques phrases sa conception du rôle d'un ministre de la culture. Plus que jamais je pense qu'effectivement il ne joue pas les modes; il est moderne.

4 Journal de F.R. 3 Charente Poitou (source: La pub mode d'emploi. F.R. 3, septembre 87). 
Candidat et tête de liste aux Législatives de mars 86, D. Bussereau, attaqué sur la même antenne par un vieux routier de la politique, a obtenu un droit de réponse: sa légitimité UDF est contestée, sa compétence aussi. D. Bussereau est très peu connu et l'attaque lui nuit d'autant plus.

Conseillé par un spécialiste en marketing politique (bien préparer son intervention, montrer la preuve de sa légitimité), le voici à l'écran lisant un texte pourtant court (feuillet visible sur ses genoux) puis déployant la lettre de soutien de Lecanuet qui le légitime lui, Bussereau, et personne d'autre. Contrastant avec son air digne, son ton courroucé et un geste déictique grondeur, ces maladresses m'amusent. Je trouve ce jeune challenger peu crédible.

Clip politique (source : La pub mode d'emploi F.R. 3., septembre 87).

7 Vidéogramme exceptionnel: M. Cenoyer, candidat indépendant aux législatives a payé et contrôlé le clip qui le met en scène.

Un texte, l'unique, sur le dernier plan: "Maurice Cenoyer, la sécurité pour tous dans les Bouches du Rhône ».

Je ne connais pas le candidat le thème m'intéresse mais le clip m'inquiète...

Quatre situations nocturnes bien typées (abords de grands cinémas, fast food, couple avec deux enfants passant dans une rue déserte, jeune femme prenant congé d'une vieille dame devant une porte cochère, toujours dans une rue déserte et peu éclairée) et chaque fois, l'oeil du maître: M. Cenoyer, se promène, nonchalant carrure imposante en légères contreplongées, et veille sur nous.

Cette sécurité dont il fait son affaire personnelle, je l'associe immédiatement à quelque chose de totalitaire. Malaise. Dans mes notes de travail, sans même y penser j'ai repéré ce clip sous l'appellation «Le Vigile».

8 Apostrophes (A2, octobre 87).

9 La meilleure émission dont pouvait rêver Rocard: le sérieux de l'enfant chéri des sondages est un stéréotype à double tranchant. Ce soir de la fin octobre, l'austère candidat potentiel continue d'affirmer (comme dans sa campagne d'affichage) que les problèmes politiques sont complexes et méritent autre chose que des shows (... les français aussi, donc).

Mais grâce au thème des livres il montre aussi qu'il est un être de chair et d'os avec une vie personnelle, un affect où les téléspectateurs dont je suis peuvent trouver matière à identification. Ses lectures éclectiques mais de qualité où le roman est omniprésent donnent lieu à des commentaires, des digressions par le truchement desquels se dessine un homme: il a "perdu" deux arbres, aime la voile (et montre qu'il s'y connait), est curieux des autres. Cet homme que l'on voit souvent grave et tendu, a été un enfant... pendant la guerre, a des enfants, a lu leurs B.D., en apprécie l'humour.

D'ailleurs il rit souvent au fil de rémission. Corps détendu, sourire, gestuelle riche, Rocard ne se crispe à nouveau que lorsqu'on revient sur le terrain miné de la conjoncture politique à quelques mois des élections.

Mais « Apostrophes », c'est avant tout Rocard côté jardin.

10 L'image d'une personnalité politique peut-elle être construite selon les stratégies que le marketing applique à des objets aussi divers qu'une entreprise, un produit commercial, une institution? Dans l'affirmative quel rôle y joue le médium télévisuel que tant de politiciens considèrent comme un nec plus ultra pour développer ou entretenir leur notoriété ? 
11 Les différents types d'émissions offrent des éclairages et des situations si variés qu'une liberté totale de choix permettrait d'organiser une campagne petit écran un peu à la façon d'une campagne publicitaire. Un passage à la télévision se prépare soigneusement et ressemble parfois à un travail de mise en scène où texte, tonalité, voire même comportement et costume sont travaillés.

12 Partons donc de l'analogie entre l'image d'un produit commercial et l'image d'un personnage politique et posons l'existence, dans le cas qui nous occupe, d'un médium privilégié, la télévision.

13 Le personnage politique s'y expose: ce sont ses situations d'énonciateur qui nous intéressent en tant que paramètres cruciaux (nous nous en expliquerons plus loin) d'une image globale à laquelle participent bien d'autres médias.

14 Précisons enfin que c'est bien de l'image d'un personnage politique qu'il s'agit et non de l'image de la politique ou de celle d'un parti. Même si, de fait, il y a contamination constante.

15 Revenons à notre postulat de départ et appliquons à notre personnage la démarche que le marketing applique à l'étude d'image.

\section{Système de Représentations et Autocensure.}

16 La réflexion porte sur cet ensemble de représentations qu'a un public d'un produit, d'une institution, d'une personne, et qui constitue l'image.

De la nature de ces représentations va dépendre l'identification et la mémorisation.

La démarche marketing vise à contrôler ces représentations pour obtenir l'image souhaitée.

Elle les regroupe en trois catégories ; appliquées au personnage politique cela donne

17 - des caractéristiques générales:

qui est le personnage? quelle est sa fonction? dans le Gouvernement? dans un parti ? quel parti?.

Quand, ouvrant l'heure de vérité en octobre 1987, F.H. de Virieu présente longuement P. Méhaignerie (Président du CDS, Ministre de l'équipement, barriste). Ce n'est pas seulement une entrée en matière rituelle: le journaliste le sait fort bien qui ajoute «Pierre Méhaignerie existe et, bizarrement, les Français ne l'ont toujours pas rencontré ». Pour nombre d'entre eux P. Méhaignerie c'est la révision du système locatif.

18 - des caractéristiques socio-politiques:

il s'agit cette fois de la situation du personnage dans le champ social et dans un champ plus étroitement politique. Itinéraire professionnel, attitudes devant des problèmes généraux de société, positionnement dans la vie politique et/ou dans le parti (pilier, nouveau leader, spécialiste, enfant terrible), actifs ou passifs dans le domaine des actions entreprises, etc...

19 - des caractéristiques personnelles :

traits particuliers de son caractère et de ses goûts personnels mais aussi aspect physique, vestimentaire, objets attributs du personnage privé et public, voix, organisation du discours, gestes et mimiques qui participent à sa fonction expressive, manière de bouger, attitudes, etc...

L'audiovisuel est bien sûr un moyen privilégié de travailler cette troisième dimension. 
20 En communication commerciale il n'est pas rare que les caractéristiques d'une image bénéficient ou souffrent de l'image du secteur professionnel tout entier.

Dans le deuxième cas des spécialistes peuvent y pallier.

En communication politique, et tout particulièrement à la télévision, l'affaire est plus délicate. La vie politique dans son ensemble, celle du parti, les avatars liés à la fonction peuvent entacher une ou plusieurs caractéristiques qui tendaient à construire une image souhaitée auprès d'un public ciblé.

21 Mais le personnage ne peut pas toujours réagir à sa guise, même si son argumentation est solide :

- ce serait plus d'une fois mettre en cause l'appareil qui le légitime.

- ce serait méconnaître les mécanismes du jeu télévisuel et de ses publics.

\section{Publics, Règles du jeu télévisuel, double Contrainte.}

22 Car même en situation favorable, construire ou renforcer une image souhaitable relève de prouesses rhétoriques dès lors que l'on ne choisit pas son public mais que l'on est exposé en même temps

23 - aux partenaires politiques :

P. Méhaignerie, Ministre de J. Chirac et barriste, n'échappe pas à la question d'A. Duhamel sur la compatibilité des deux positionnements et doit ménager R. Barre et le Premier Ministre,

24 - aux opposants (ceux qui existent vraisemblablement à l'intérieur du groupe ou ceux d'autres partis) :

à ces censeurs il ne faut pas prêter le flanc. Une image malheureuse (renvoyer R. Monory à son garage) employée par L. Jospin à l'Heure de Vérité est rappelée dans l'émission suivante où $P$. Méhaignerie y souligne une signification idéologique peu compatible avec les valeurs revendiquées par le P.S.

25 - à la presse :

elle donnera une lecture de l'émission qui n'est pas contrôlable par l'énonciateur. Et puis, bon gré mal gré, des contraintes commerciales l'incitent souvent à amplifier la portée d'un propos et, à plus forte raison, d'un mot malheureux.

26 - aux groupes d'actualité (ceux qui sont concernés par tel sujet sur lequel l'énonciateur a prise) :

P. Méhaignerie évoque le projet destiné à aider les mairies pauvres, D. Bussereau s'adresse aux chefs d'entreprise bons relais d'opinion, J. Lang rappelle les secteurs culturels peu traditionnels qu'il s'est employé à promouvoir etc...

27 A ces relais d'opinion s'ajoutent

28 - les militants :

les apparatchiks ou militants de base sont sensibles au moindre démarquage,

29 - le grand public:

il n'est pas sensible au même titre que les autres publics à la prestation elle-même, mais, à côté des opinions arrêtées et des indifférents, le personnage politique sait qu'il y a des opinions flottantes, celles des indécis qui, ni pour ni contre, regardent, écoutent et sont en situation d'être influencés favorablement.

Ils sont au cœur de la cible. 

l'énonciateur. Travailler une image c'est agir sur une préexistence minimale dans le public ciblé (D. Bussereau est un tout petit notable, «le Vigile » récupère des images d'extrême droite) qu'il s'agit de développer et/ou de modifier et/ou de nourrir régulièrement afin qu'elle soit, comme en communication commerciale, perceptible et désirable ${ }^{1}$. minimum d'harmonie avec un groupe ou des institutions qui, au fil de l'actualité politique, ne servent pas toujours cette image? Comment dénoncer des stéréotypes sociaux ambiants sans risquer de déstabiliser les individus, et du même coup de devenir ou de rester non désirable? Il y faudrait toutes les ressources de l'« inventio » rhétorique .

Or, sauf en temps de campagne présidentielle les règles du jeu des émissions n'y sont pas favorables : derrière le petit écran les contraintes audiovisuelles (durée de l'émission, interfocuteurs, découpage en thèmes, répartition des temps de parole, etc...) limitent l'énonciateur dans son argumentation ou ses possibilités de contre-argumentation.

Et ce n'est pas un hasard si de l'autre côté de l'écran on trouve des téléspectateurs qui, même s'ils sont en situation d'être influencés, n'en appliquent pas moins la loi du moindre effort: l'information, oui, mais avec des facilitateurs, de la distraction, du spectacle. compenser par d'autres moyens.

On verra à l'instant que le médium télévisuel y devient fécond.

\section{L'Actio au secours de l'inventio.}

En communication commerciale les messages de base (« le choix », « le bon marché », « le service après vente») sont désormais des positionnements physiques insuffisants: d'autres les revendiquent et il faut se différencier.

Dresser une argumentation complexe pour faire valoir que telle notion de choix est préférable à telle autre serait aussi suicidaire (la cible en communication commerciale n'est pas moins paresseuse qu'en communication politique) que de vouloir en politique culturelle, « démontrer » où se trouve le « mieux-disant ».

Mieux vaut le «montrer» autrement. Cet autrement donne lieu en image de marque commerciale à un prisme d'identité où, au positionnement physique s'ajoutent cinq autres facettes conçues pour déclencher l'adhésion à un produit personnifié, via la séduction.

- La facette personnalité correspond au tempérament attribué au produit par le récepteur,

- la facette relationnelle correspond au rapport de distance, étrangeté, complicité perçu par le récepteur,

- la facette culturelle renvoie aux valeurs culturelles attachées au produit par le récepteur,

- la facette reflet correspond à l'image que le récepteur pense donner de lui en adoptant le produit,

- la facette mentalisation correspond à l'image que le récepteur aurait de lui-même en adoptant le produit. 
Ainsi à titre d'illustration sommaire, et avec des variantes non négligeables d'une marque à l'autre, tout un segment du marché de la bière s'installe sur un prisme d'identité novateur ${ }^{3}$ où la personnalité du produit devient raffinement des hommes et des femmes, où la facette relationnelle évoque la complicité ou la convialité urbaine plutôt que la cordialité virile autour d'une mousse ; à la place du terroir, du travail, de loisirs masculins traditionnels, la facette culturelle recherche valeurs artistiques, coktails, soirées chic, ambiance jeune des brasseries branchées, érotismes diffus, ambigus. La facette reflet qui en découle fonctionne au travers d'associations d'idées installées par l'idéologie que nous venons d'évoquer et la facette mentalisation, via les possibilités d'identification.

Pour travailler une image de personnage politique à partir du prisme d'identité et notamment des facettes relationnelles, culturelles et de personnalité, la télévision est un canal privilégié : l'exposition physique de proximité (plans de demi-ensemble, nombreux plans rapprochés, gros plans) où le personnage est tout à coup non plus seulement une voix, ou une série de réponses déformées par l'écrit, ou un discours indirect... mais un corps évident - qui se voit $-{ }^{4}$, favorise les performances énonciatives, la fonction expressive du verbal, toutes celles (référentielle, métalinguistique, expressive, phatique, conative, poétique) du non verbal et, s'adressant à l'affect, devient susceptible de déclencher l'adhésion.

41 Le canal télévisuel s'illimite les ressources de l'«inventio » peut donc rehausser sa crédibilité grâce à son interaction avec deux autres aspects de la rhétorique, l'actio (le passage à l'acte de discours) et l'elocutio (le style de discours).

\section{Limites de l'Actio : Quand le corps résiste.}

Nous venons d'écrire "peut rehausser sa crédibilité »... Pour expliciter cette réserve revenons un instant à nos facettes.

Pour que les facettes «reflet» et «mentalisation» aient quelque chance de susciter l'adhésion d'un récepteur en situation d'être influencé, il nous paraît clair que les autres facettes doivent non seulement lui plaire une à une mais lui offrir encore une cohérence d'ensemble satisfaisante. Pas toujours facile pour l'image politique !

En communication commerciale une image se construit autour d'un produit créé de toutes pièces ou ajusté; des spécialistes font "parler» leur produit en fonction des « lectures », des représentations souhaitables c'est-à-dire propres à entraîner l'adhésion.

En communication politique une image se construit dans des conditions plus difficiles. Le corps est un produit préexistant. Certes, on peut l'ajuster (coiffure, vêtements, accessoires, environnement, ...) plus ou moins subtilement pour faire "parler» ce signifiant raconté par la presse, captif bon gré mal gré des objectifs, en fonction des «lectures" souhaitées ${ }^{5}$.

45 Mais sous ces ajustements de surface il y a un corps qui résiste, qui parle un langage « sui generi ». Avec deux aspects :

- des caractéristiques physiques (corpulence, traits, timbre de la voix, etc.) qui ne peuvent être changées.

Et là se dessine pour l'image de marque un premier obstacle possible : peut-on contrôler des lectures fondées sur des stéréotypes culturels profondément ancrés?

Le « Sphinx » Mitterrand aurait-il le même impact si, par commutation physique, il avait le port de tête, la carrure et les traits d'un M. Jobert? 
(Il y aurait une enquête réjouissante à mener sur la crédibilité accordée au corps des femmes politiques).

- des caractéristiques expressives (réunissons provisoirement sous ce terme tout ce qui relève de l'actio et de l'elocutio en situation d'improvisation).

Nombre de caractéristiques préexistantes, intégrées, peuvent être développées et adaptées au médium télévisuel ${ }^{6}$. Mais quand il s'agit de contrôler, voire de juguler des caractéristiques jugées négatives pour l'image de marque, on rencontre les obstacles de la résistance et de l'oubli. «Oubli»-M. Rocard sait probablement qu'il doit être attentif à son élocution. Qu'il l'oublie un instant et le débit se précipite en fin de phrase, provoque une gêne passagère et brouille la compréhension.

Le corps est têtu, il « trahit son homme ». Son entraînement reste ingrat et expose même le personnage à des dissonances préjudiciables à l'adhésion souhaitée chez le récepteur.

« Résistance » - J. Chirac a beau essayer de préserver l'allure dynamique qui le sert tout en gommant les traits agressifs: des gestes heurtés, la contraction des mâchoires trahissent fréquemment sa tension intérieure et peuvent contredire les propos tenus.

Un découpage très fin des plans montrerait pour nombre de prestations de personnages politiques, des gestes, des mimiques qui ne parlent pas le même langage que le verbe. "Assis entre deux chaises »- Début novembre à "L'heure de Vérité », P. Méhaignerie assure qu'il vit tout à fait bien une double situation (ministre de J. Chirac et barriste à la fois) qui ne serait paradoxale qu'en apparence.

Pourtant, ses gestes (mains en couvercle, comme pour contenir les faits évoqués, déictiques en parallèle de part et d'autre du corps notamment) disent le contraire.

Bien entendu, oublis, résistances, dissonances ne se traduisent pas systématiquement par des effets d'incohérence jugés « soupçonnables » par le récepteur.

Mais avec des procédures comparables à celles appliquées par Jon Baggaley et Steven Duck ${ }^{7}$ il serait intéressant d'expérimenter comment une énonciation qui présenterait des variantes dans l'actio serait susceptible de provoquer des niveaux d'adhésion différents chez un récepteur en situation d'être influencé.

\section{Morale du corps et Marketing.}

54 Importance de l'actio pour l'image du personnage politique dans un monde médiatisé où le petit écran est un paramètre de choix, soit.

Importance du développement des qualités médiatiques chez un personnage politique, soit.

Mais, si ces vertus peuvent aider à compenser une argumentation trop complexe pour le médium télévisuel, les hommes politiques et les conseillers en communication devraient se demander régulièrement si l'actio peut compenser :

- une absence d'argumentation,

- une argumentation incohérente,

- des non-dit pesants (raison d'État, stratégies politiciennes, etc.),

- un manque d'adhésion de l'énonciateur à son propre discours.

Autrement dit, le personnage politique a beau accepter de se prêter à un entraînement visant une meilleure communication, mieux vaut ne pas le pousser aux rôles de composition. 
Ce n'est pas forcément son fort. Surtout quand le scénario politique prend des tours imprévisibles.

\section{BIBLIOGRAPHIE}

Barthes, R., (1970), « l'Ancienne Rhétorique », Communications, 16, Seuil.

Porcher, L., (1976), « Introduction à une sémiotique des images », Didier.

Reboul, O., (1984), « la Rhétorique », Coll. Que sais-je ?, PUF, pp. 18 à 28.

\section{NOTES}

1. Le marketing d'image ajoute au "perceptible» et au "désirable» une caractéristique « différentielle»; nous l'avons jugée sous-jacente au " perceptible».

2. Pour les notions empruntées à la rhétorique antique tout au long de ce texte, voir O. Reboul, «la Rhétorique», Coll. Que sais-je?, PUF, 1984 (pp. 18 à 28) et R. Barthes, "l'Ancienne Rhétorique ", Communications, 16, Seuil, 1970.

3. Il s'agit en fait d'une série de prismes dont la réussite tient à des oppositions pertinentes et dont nous ne présentons ici que « l'archiphonème » approximatif.

4. Bien entendu ce corps qui se voit est une image fortement médiatisée où les manipulations (angles de prise de vues, éclairages, cadrages, montage, etc) donnent lieu à des variations d'effets de sens patents. Voir notamment les travaux d'E. Véron et l'article de Jon Baggaley et Steven Duck, « La crédibilité du message télévisuel », Communications, 33, Seuil, 1981.

5. Cf. L. Porcher, «Introduction à une sémiotique des images ", Didier, 1976, où l'auteur expérimente sur un corpus d'images publicitaires les effets de lecture produits par des commutations d'items icôniques.

6. Nous pensons ici aux potentiels naturels qui peuvent être développés par le milieu familial et social, l'enseignement, puis des moments de formation spécialisée.

7. Cf. note 4 .

\section{RÉSUMÉS}

La télévision a bouleversé l'image des personnalités politiques. Celles-ci ont dû intégrer dans leurs stratégies les notions de communication politique et de marketing. L'homme politique est devenu un produit que l'on cherche à vendre. L'auteur explique les différents ressorts de ce marketing politique et l'importance des publics et de leurs réception et compréhension de ces phénomènes dans ce domaine. 
INDEX

Mots-clés : communication politique, télévision, sémiologie, marketing politique

Keywords : political communication, television, semiology, political marketing

\section{AUTEUR}

ODILE AUSINA

Odile Ausina, Université de Lille 3/CERTEIC 